\title{
Coming of age
}

\author{
As Nature Photonics turns 10 years old, we reflect on how times have changed and offer a message of \\ thanks to our authors, reviewers and readers.
}

On 21 December 2006, the inaugural issue of Nature Photonics - January 2007 went live on our website. Now, 10 years later we have cumulatively published over 8,400 pages of editorial content, including more than 800 Articles and Letters and almost 50 supplements and focuses. Over that period, we have received a total of over 9,000 submissions to the journal and have seen average submissions steadily grow to $\sim 100$ submissions per month.

While it is tempting to reminisce over individual papers that we have published in the past decade, we would prefer to make a general comment about the diversity of the research that has found its way into the pages of Nature Photonics. Findings have spanned the electromagnetic spectrum from X-rays to terahertz waves, targeting a myriad of applications ranging from quantum information processing to superresolution imaging. Furthermore, some of these papers, most notably on the topics of organic solar cells and hard X-ray generation by free-electron lasers, have received over 1,000 citations to date.

To celebrate our tenth birthday, in place of the usual News \& Views section, this issue of Nature Photonics contains a special set of eight commissioned forward-looking features in the areas of solar cells, quantum optics, imaging, terahertz science, $\mathrm{X}$-rays, optical communications, organic optoelectronics and plasmonics written by experts in their respective fields. These are complemented by a Books \& Arts piece featuring a selection of our all-time favourite books in photonics.

It is clear that the success of Nature Photonics has gone hand-in-hand with the incredible achievements and evolution of the photonics sector itself. Photonics undoubtedly now has far greater prominence and activity than ever before. It has truly become a pervasive science and a widely recognized enabling technology platform.

Since we launched, scientific breakthroughs with a strong connection to photonics have been directly rewarded by no less than five Nobel Prizes for Physics and Chemistry for achievements related to quantum optics, imaging, optoelectronics, optical communications and light sources (see page 11 for a visual representation). And while the recent announcement of the detection of gravitational waves at the Laser Interferometer

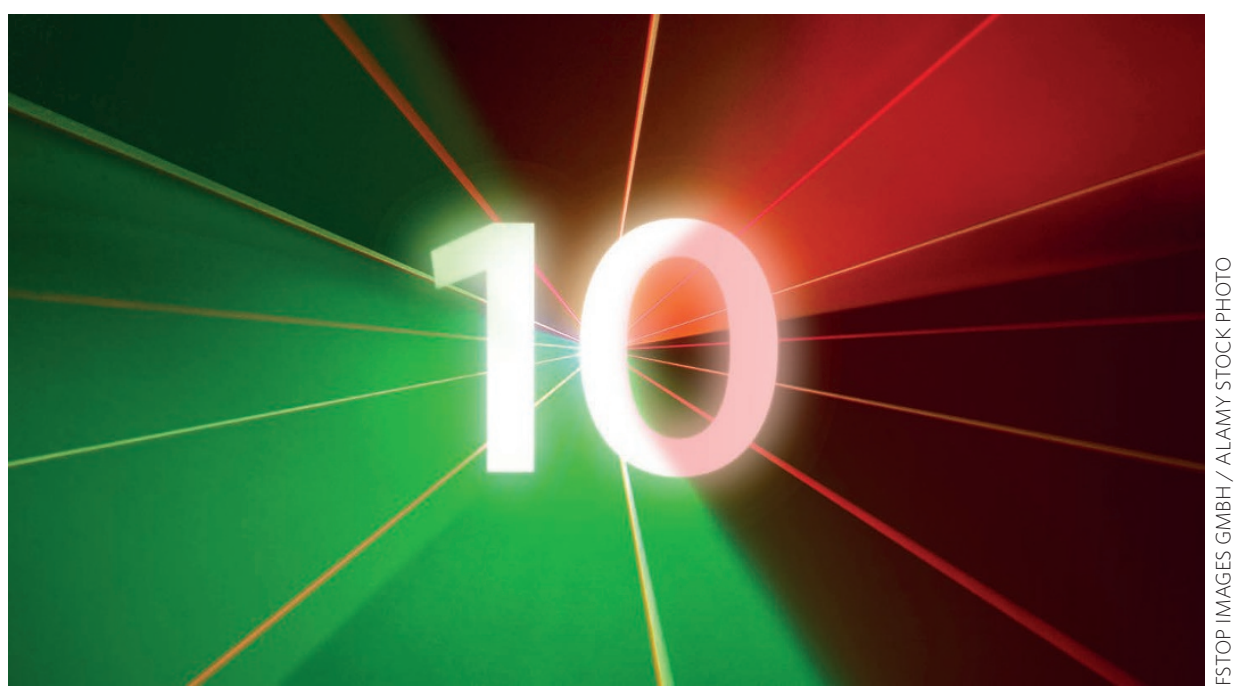

Gravitational Wave Observatory (LIGO) in the US in February 2016 has not received a Nobel Prize yet, photonics played a pivotal role in the sensational discovery.

In 2015, UNESCO (United Nations Educational, Scientific and Cultural Organization) recognized the importance and impact of photonics with its International Year of Light (www.light2015.org), and an extensive series of 13,000 events around the globe promoting education and celebration of photonics took place. The important role that photonics can play in tackling pressing societal challenges such as energy generation (see photovoltaics feature on page 3),

reducing energy consumption in lighting and communications (see optical communications feature on page 5) and improved healthcare (see imaging feature on page 14) is now widely acknowledged.

In Europe, Photonics 21 (www.photonics21.org), an umbrella organization for research and development in both academia and industry, now has more than 2,500 members and has ensured that photonics is well represented in the European Union's Horizon 2020 funding programme. While in the US, the National Photonics Initiative (www.lightourfuture.org) was established in 2013 by a consortium of US societies to identify, support and advance photonics deemed of strategic importance to the US economy. A recent report from France's Directorate General for Enterprise estimates that the world market for photonics exceeded US $\$ 481$ billion in 2012 and will reach US $\$ 620$ billion by 2020 , a growth rate of nearly $30 \%$ (go.nature.com/2hEiWSc).

More researchers than ever before are now performing research into photonics and publishing an ever expanding quantity of findings in a greater number of journals dedicated to the field. In the past few years, the number of optics journals has grown dramatically with the launch of a raft of new titles from acclaimed publishers such as ACS Photonics from the American Chemical Society, Optica from the Optical Society of America, APL Photonics from the American Institute of Physics and Light: Science \& Applications by a partnership between Changchun Institute of Optics Fine Mechanics and Physics (CIOMP) and Springer Nature. The UK's Institute of Physics has also relaunched its Journal of Optics. Scientists now have more choice than ever before as to where to publish their research and we welcome this freedom.

It's fair to conclude that photonics has truly come of age and the future is bright. Despite all these developments, the mission of Nature Photonics to publish impactful, high-quality research in the sector remains unchanged. This is not possible without the support of the optics community and we wish to offer our sincere thanks to those of you that have submitted papers and reviewed for us since our launch. It is much appreciated. We look forward to working with you all over the next 10 years. 